\title{
Statistical and Wavelet Analysis of the Solar Wind Data
}

\author{
Maurício José Alves Bolzan \\ Laboratório de Física Atmosférica e Solar, Instituto de Pesquisa e Desenvolvimento, Universidade do Vale do Paraíba, UNIVAP \\ Received on 11 March, 2004. Revised version received on 15 October, 2004
}

\begin{abstract}
We perform statistical and wavelet analysis of three time series based on the solar wind velocity of the year 2000, the original time series and two filtered components. We use the Haar Wavelet Transform to separate this annual time-series into two parts, corresponding to high and low frequencies. We then calculate the kurtosis and skewness parameters for the three time-series. The results show that these parameters present high values in the low-pass filtered time-series, indicating that the intermittence level is superior at large scales (low-frequencies) if compared with the small scales of the turbulence. It is possible to conjecture that the Coherent Structures are responsible for this behavior. The use of the Morlet Wavelet Transform is presented to understand this behavior in terms of the gradient and nonlinear interaction of energy between scales.
\end{abstract}

\section{INTRODUCTION}

The study of geomagnetic indices, solar wind velocity and proton density in solar-terrestrial relations and magnetospheric physics is very important due to several activities on Earth, as data transmission by satellite, working of power lines, and many others. Thus, the study of the statistical properties of geomagnetic indices and their relations to the solar wind has attracted a growing interest $[1,2]$. According to Hnat [3], statistical properties of velocity field fluctuations recorded in wind tunnels or obtained from solar wind observations exhibit striking similarities. The common feature in these fluctuations is the statistical intermittence. The intermittence phenomena opened up an interesting research area. Kinney and collaborators [4], investigating the phenomenology of magnetohydrodynamic turbulence through of direct numerical simulations, have established the existence of Coherent Structures (CS) called coherent magnetic vortices. These vortices control the turbulent cascade of indirect current sheets generated during close encounters between the vortices. Furthermore, the energy spectra have a time dependence at early times, which is associated with coalescence events of the CS. Hussain [5] defined the CS in turbulent variables (velocity, temperature, density and others) that have high self-correlation or other variables in the determined time scale. The structures are strongly associated with energy dissipation of the turbulent flows and also a source of instability at least in some scales. In this work, we will use the statistical analysis to study the intermittence and the CS that are present in experimental data. We will calculate the skewness, which is a less explored parameter in magnetohydrodynamics turbulence, and the kurtosis for high-pass and low-pass filtered time series of the solar wind velocity. In order to filter the time series, we will use the Haar Wavelet Transform to decompose it in all possible frequencies. Furthermore, we will apply the Morlet Wavelet Transform and Global Wavelet Spectrum in order to understand the behavior of energy between large and small scales.

This paper is organized as follows. Section two is devoted to the theoretical background of statistical and wavelet analysis. Section three is concerned with the measurements and the experimental site. In the fourth Section, we present some results and discuss their consequences. Finally, in last Section, we present some conclusions.

\section{THEORETICAL BACKGROUND}

\section{A. Statistical Analysis}

Due to the difficulties to study turbulence in computer simulations, statistical analyses as the Probability Density Functions (PDF) are useful tools to understand the phenomena that occur in any turbulent system, involving hydrodynamic [6,7] or magnetohydrodynamic $[8,9]$ flows. Two statistical parameters that have been used to accomplish this objective, the skewness and kurtosis parameters.

According to Frisch [10], the kurtosis is a measure of intermittence in flows and is the parameter that controls the shape of PDF. This parameter is calculated from the relation

$$
K_{r}=\frac{\left\langle v_{r}^{4}>\right.}{\left\langle v_{r}^{2}\right\rangle^{2}},
$$

where $r$ is the increment scale and $v_{r}(x)=v(x+r)-v(x)$.

The skewness parameter was used to investigate the transport asymmetry in hydrodynamics turbulence, specifically in Convective Boundary Layer (CBL) in atmosphere [11]. In recent years, there are many works demonstrating the importance of the study of the skewness parameter in magnetohydrodynamics turbulence data obtained in laboratory [12] and experimental sites [13].

\section{B. Wavelet Analysis}

The Fast Fourier Transform (FFT) is a useful tool to study the power spectrum of stationary time series. A distribution of spectral density identifies the energy associated with the frequencies, but does not give any information about temporal localization in the time series. In other words, FFT is a global transform. For a signal $x(t)$, the Fourier transform is given by

$$
X(f)=\int_{-\infty}^{\infty} x(t) e^{-2 j \pi f t} d t
$$

This analysis, however, is not adapted to non-stationary timeseries. In order to overcome this difficult, Morlet developed the Wavelet Transform (WT). Meyer [14] demonstrated the conditions for orthogonality of this new mathematical operator. The wavelet word indicates a set of functions with the 
form of small waves created by dilations, $\Psi(t)=\Psi(2 t)$, and translations, $\Psi(t)=\Psi(t+1)$, from of simple generator function, $\Psi(t)$, which is called mother-wavelet. Mathematically, the wavelet function with a scale $a$ and at position $b$ is given by

$$
\Psi_{a, b}(t)=a^{-1 / 2} \Psi\left(\frac{t-b}{a}\right)
$$

where $a$ and $b$ are real and $a>0$. The Wavelet transform is defined by

$$
\left(W_{\Psi} f\right)(a, b)=\frac{1}{a^{1 / 2}} \int f(t) \Psi\left(\frac{t-b}{a}\right) d t,
$$

where the temporal function $f(t)$ is any time series.

Exist two types of wavelet functions, the continuous and discrete wavelets. The most famous discrete wavelet families are, Haar wavelet [15], Meyer and Daubechies [16], while the most famous wavelet families are, Morlet [17] and the mexican-hat [18].

In this work, we used the two types of wavelet function, the Morlet wavelet applied to a time series for investigating the dominant period, and the Haar wavelet, for filtering the time series into two parts, one part with high frequencies only, and another one with low frequencies only.

\section{DATA}

In this work we used data of solar wind from SOHO satellite obtained in the public internet address http : //umtof.umd.edu/pm/crn/ [19]. Data were obtained during the year 2000, with a sampling rate of one measurement by hour (approximately $2.78 \times 10^{-4} \mathrm{~Hz}$ ). This paper deals with the analyses of the solar wind records in this year. Graphs in Fig. 1 show the data set for 12 months of this year.

The curve represents a characteristic scenario in the evolution of the solar wind, notably the rapid change of the magnitude of solar wind velocity in July, where the increase in magnitude is associated with the very strong solar disturbance that occurred in this year. Kovács [1] illustrated that if one disregards the real reason for the evolution of the geomagnetic disturbances (storms, or substorms), the fluctuating nature of the field can be interpreted in the present context as the manifestation of turbulent phenomena that take place within the plasma of the magnetosphere. It has long been accepted that turbulence evolves through cascade processes that involve a hierarchy of coherent vortex structures belonging to a wide range of spatial scales. Kolmogorov [20] proposed the inhomogeneous flow down (cascade) of the energy from system-size scales to dissipative (Kolmogorov) ones. The inhomogeneity involves the singular behavior of the energy distribution in physical space resulting in strong gradients, or intermittence in the time-series of the energy related to physical quantities of the system, e.g. velocity [21], temperature [7] or magnetic fields $[1,2]$. Thus, we used the approach of this Kolmogorovian scenario to study this data.

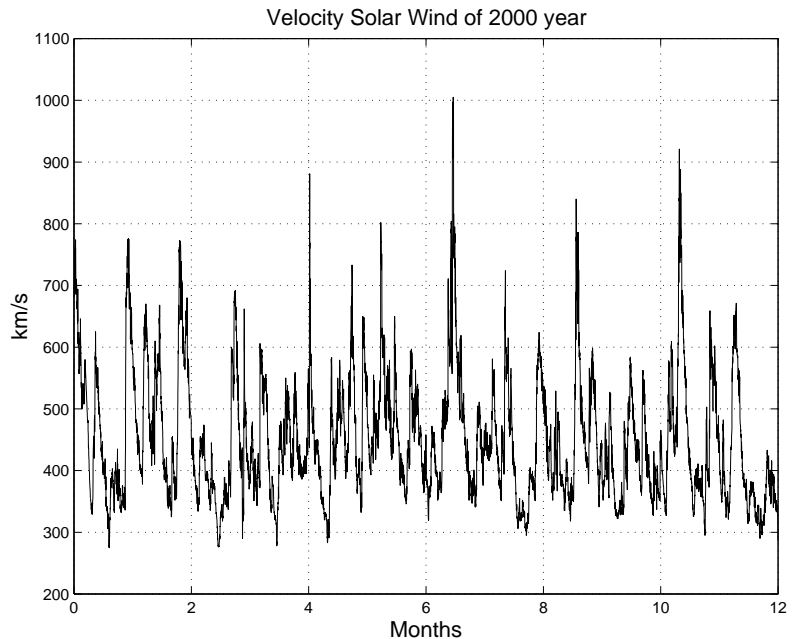

FIG. 1: Solar wind velocity time-series obtained by SOHO satellite at year 2000 .
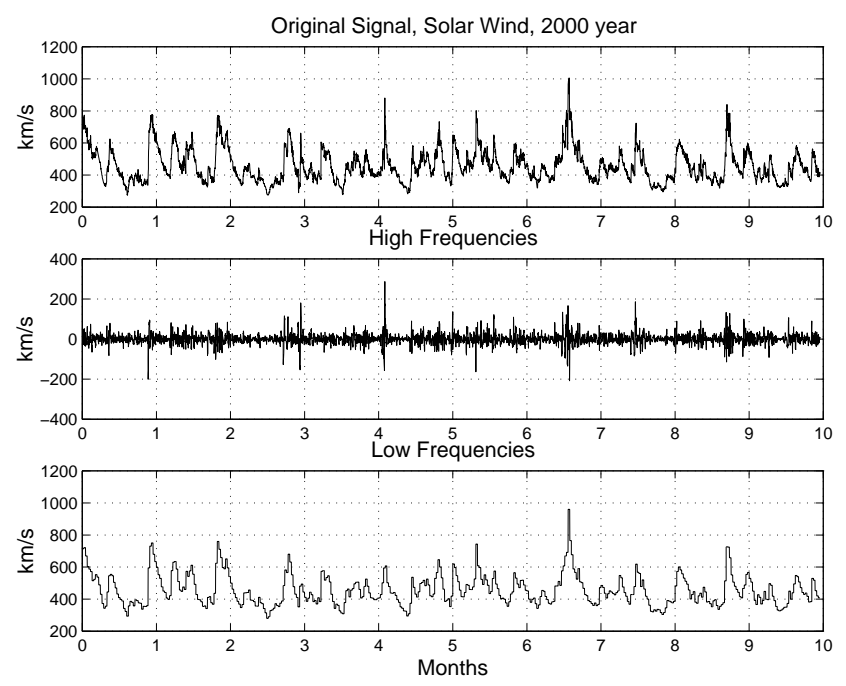

FIG. 2: Decomposition of time-series of solar wind in two parts through of the Haar wavelet transform.

\section{RESULTS AND DISCUSSIONS}

In order to perform a more detailed investigation of intermittence and coherent structures we decompose the time series into two parts. The first time series presents high frequencies only. The other time series presents low frequencies only. For this purpose, we used the Haar Wavelet Transform, with a cut-off frequency of $2.17 \times 10^{-6} \mathrm{~Hz}$. This frequency choice was obtained from the visual aspect of all of the frequencies that are present in the time series. Fig. 2 shows the results of this decomposition.

The next step was to calculation of the kurtosis parameter given by equation (1), with 30 increment scales $r$ for the two filtered and the original time series. According to Frisch 


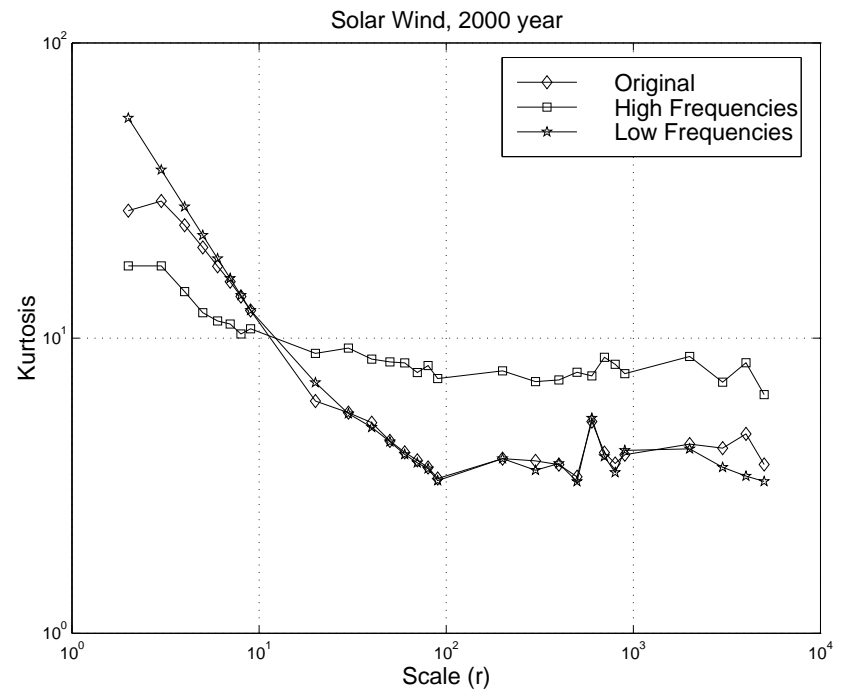

FIG. 3: Kurtosis parameter calculated in 30 increment scales to three time-series, velocity solar wind, high-pass filtered velocity solar wind and low-pass filtered velocity solar wind.

[10], the kurtosis is a measure of intermittence in flows. Large values of the kurtosis has also been observed in strongly nonGaussian Probability Density Functions (PDFs) $[3,4,6,7,8,9]$. In Fig. 3, we observe that the intermittence level grows with decreasing values of the $r$ increments for the three time series. For $r \rightarrow 0$, the values of kurtosis parameter grow exponentially, reaching maximum values in the low-pass filtered time series. Furthermore, we observe that the three time series are self-similar [9] at large scales, i.e., the kurtosis parameter shows constant values for determined $r$ intervals. The results for low-pass filtered time series are in contrast with results obtained by Ramos [23] for data referring to the Amazon forest. In this work, the high-pass filtered data showed an increase of the kurtosis parameter, exposing the intermittent character of high frequencies. In the present work, this result shows that the intermittence and coherent structures are present in the large scales of the time-series, contrary to the results of Ramos [23]. However, it is important to note that the actual results and the results obtained by Ramos [23] are different from the point of the used data, since the data of Ramos [23] were obtained in a situation of hydrodynamic turbulence while the data used in this work were obtained under magnetohydrodynamic turbulence. Also, according to Biskamp and Schwarz [24], although both kinds of turbulent flows have similar cascade properties, they do not always display the same statistical behavior.

According to the behavior studied above, it would be possible to conjecture that the differences between both time series can be directly associated with the large number of solar storms in 2000. Thus, with the purpose of analyzing this difference between both filtered time series, we applied the Morlet Wavelet Transform for constructing a periodogram. The periodogram is a tridimensional graph in which the $\mathrm{x}$-axis denotes the current time of duration of the time series, and the $y$-axis denotes the frequencies or periods in the time series; the gray scale bar represents the third dimension of the periodogram, and denotes the energy associated with each frequency or period. In Fig. 4a, we show the periodogram obtained for a high-pass filtered time series, and in Fig. 4b we show the periodogram obtained for a low-pass filtered time series. Figs. 4c and d show the Global Wavelet Spectrum (GWS) [25] for each time series. From the analysis of the periodograms, we note that the high-pass filtered time-series, Fig. $4 \mathrm{a}$, presents more defined and compact structures than the low-pass filtered time-series, Fig. 4b. This behavior can be interpreted according with to the Heisenberg uncertainty principle, i.e., in the high-frequency region we observe that the structures concentrate in a small time interval ( $\mathrm{x}$-axis) but are dispersed in terms of periods (y-axis). This behavior is the opposite of Fig. 4b, in which we observe that structures are dispersed at large time intervals, but are concentrated in a few period intervals. Thus, we write the Heisenberg principle as $\Delta t \Delta P \sim$ constant, where $\Delta t$ is the time interval ( $\mathrm{x}$-axis) and $\Delta P$ is the period interval (y-axis). The constant in this relation can be associated with the energy of each structure. This results has important physical features. At high frequencies, the structures associated with intermittent events try to dissipate energy at all possible frequencies, according of Kolmogorov's cascade. It should be pointed out that this conclusion is just a new form of definition of intermittence.

Analyzing the energy of both time-series through of GWS, Figs. $4 c$ and $4 d$, we note that the energy of the high-pass filtered time series has a small contribution to the time series as compared with the low-pass filtered time series, Fig. 4d. These results show that the energy from large scales can be useful for the creation of the coherent structures at large scales (low frequencies). The larger values of the kurtosis parameter at the large scales may be an indication of the intermittence phenomena caused by the CS.

In order to study the CS, we used the skewness parameter. The histogram of solar wind velocity presents a strong skewness [26], centered at $400 \mathrm{kms}^{-1}$, as shown in Fig. 5. This fact shows the presence of gradients in this time series. As in the treatment of the kurtosis, we calculated the skewness parameter with 30 increments in the $r$ scale in the two filtered and original time series. Fig. 6 shows the results. We note that the skewness alternates between positive and negative values for large values of the $r$ parameter, but it becomes positive along the direction of the small values of the $r$ increment parameter. Furthermore, we observe that the large scales contribute strongly for positive skewness. In general, the kurtosis and skewness parameters have the same behavior, also according recent results of Burgala and Forman [13]. Thus, these results shows that the source of skewness of the large scales is due the presence of the coherent structure in the time series. However, in this study it is not possible to determine the geometrical configuration of these structures. Also, in future works we plan to analyze another time series in order to see whether a similar behavior still occurs in the years of high and low solar activities. 

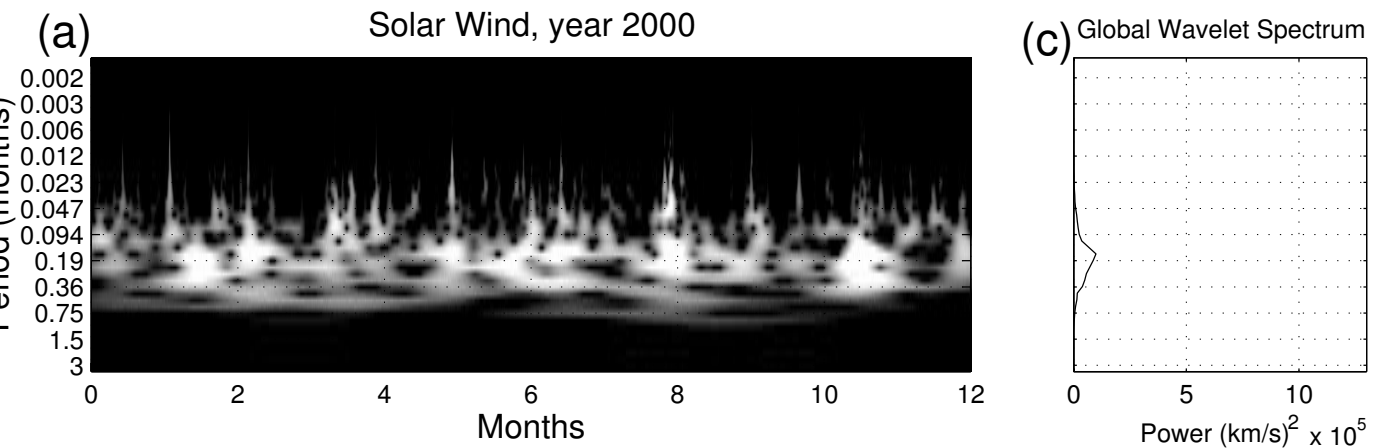

(b)

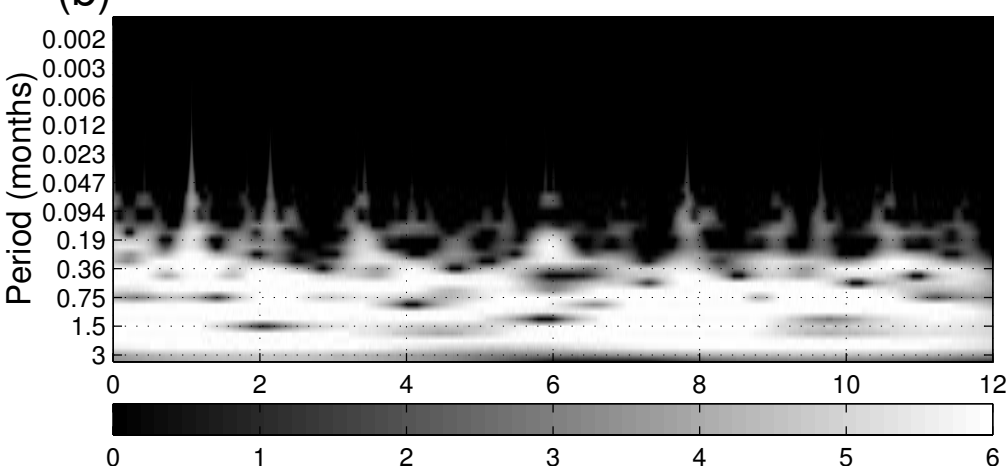

(d)

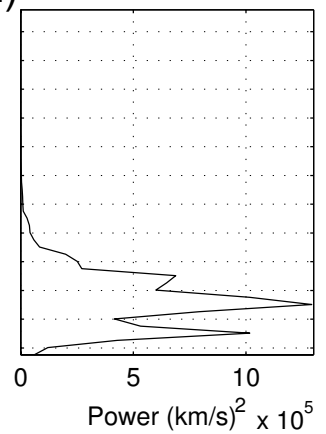

FIG. 4: a) Periodogram of Morlet Wavelet Transform applied to time-series of solar wind of the year 2000 high-pass filtered; b) Periodogram for time-series low-pass filtered; c) Global Wavelet Spectrum for time-series high-pass filtered and; d) Global Wavelet Spectrum for time-series low-pass filtered.

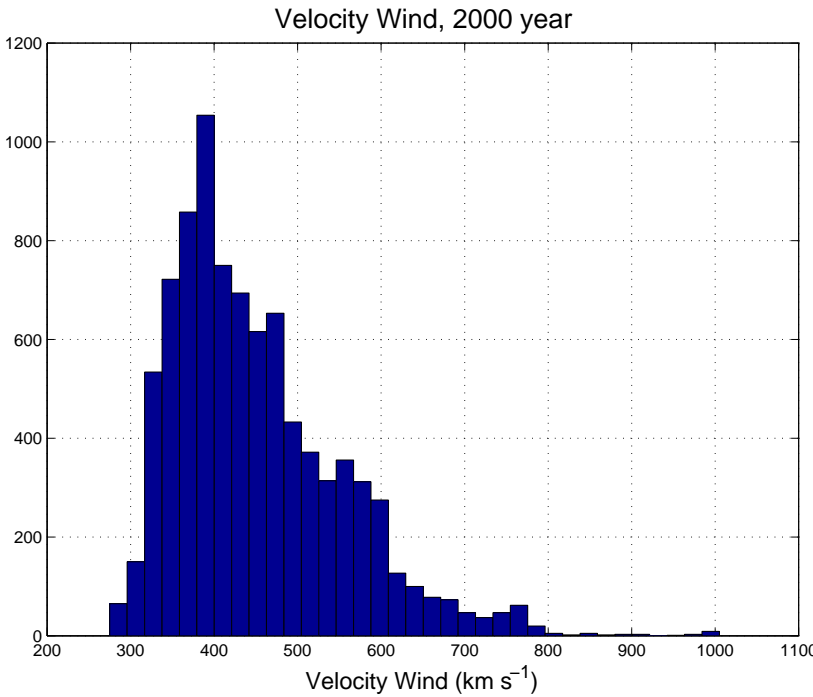

FIG. 5: Histogram of velocity solar wind, year 2000.

\section{CONCLUSIONS}

In this work, we used the annual time series of the solar wind velocity, measured by the SOHO satellite in the year 2000, in order to study the presence of Coherent Structures and intermittence phenomena. We used the Haar Wavelet Transform to separate this annual time series of the solar wind velocity into two parts, one part of high frequencies and another one of low frequencies. We then used a statistical analysis for calculating the kurtosis and skewness parameters.

Results for the kurtosis have shown larger values in the lowpass filtered time-series as compared with other time-series with the $r$ interval between $10^{0}$ and $10^{2}$, showing the higher level of the intermittence in the large scales of the flow. This result shows that the intermittence level is higher at the large scales as compared with the small scales of the turbulence. It is possible to conjecture that the source of this intermittence are the Coherent Structures at the large scales.

In order to further understand these phenomena, we studied the behavior of the skewness parameter for the three time series. The results have shown that the low-pass filtered time series also presents large values of the skewness as compared with the other time-series. Furthermore, we used the Morlet Wavelet analysis and Global Wavelet Spectrum to study the energy interaction between scales. The results for the 


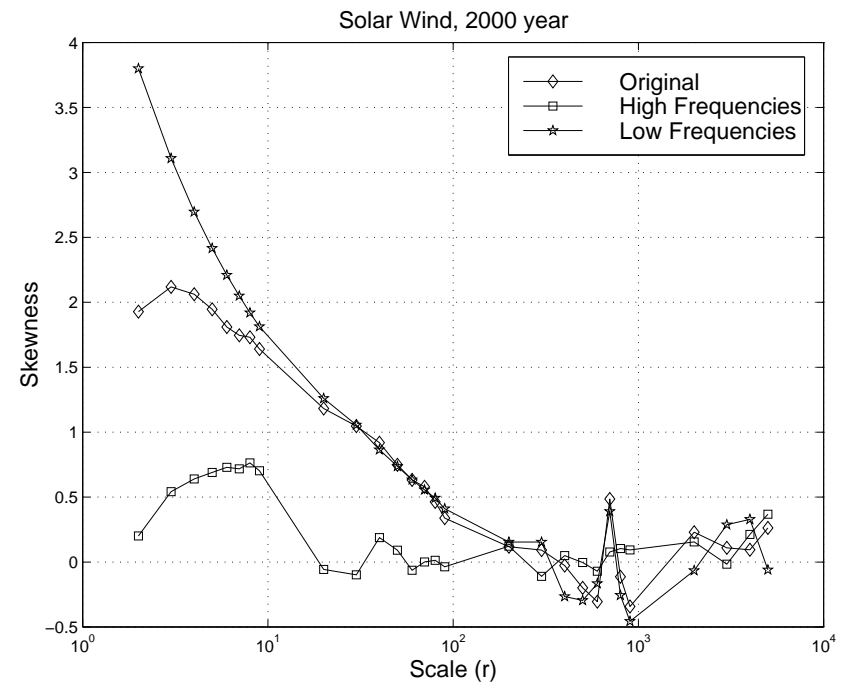

FIG. 6: Kurtosis parameter calculated in 30 increment scales to three time-series, velocity solar wind, high-pass filtered velocity solar wind and low-pass filtered velocity solar wind. low-pass filtered time-series showed strong energy at the large scales.

These results lead to the following physical conjecture. The high energy at the large scales of the magnetohydrodynamics turbulence, due the high solar activity in 2000, contributes to creation of Coherent Structures in the time series. Furthermore, based on the results for the kurtosis and skewness analyses, we conclude that the solar wind velocity presents a space gradient along the distance between the Sun and the Earth, and that this gradient can be associated with the solar activity. These conclusions are consistent with the results from statistical and wavelet analysis for hydrodynamics turbulence performed by Katul [27].

\section{Acknowledgements}

We thank the referees for fruitful comments and suggestions.
[1] P. Kovács, V. Carbone, and Z. Voros. Planetary and Space Science, 49, 1219 (2001).

[2] A. T. Y. Lui. Journal of Atmospheric and Solar-Terrestrial Physics, 64, 125 (2002).

[3] B. Hnat, S. C. Chapman, and G. Rowlands. Intermitency, scaling and the Fokker-Planck approach to fluctuations of the solar wind bulk plasma parameters as seen by wind. Phys. Rev. E, 67, 5 (2003).

[4] R. Kinney and J. C. McWilliams, Physic of Plasmas, 210, 3623 (1995).

[5] A. K. M. F. Hussain. Physics of Fluids, 26, 10, 303 (1983).

[6] F. M. Ramos, R. R. Rosa, C. Rodrigues Neto, M. J. A. Bolzan, and L. D. A. Sá . Physica A, 295, 250 (2001).

[7] M. J. A. Bolzan, F. M. Ramos, L. D. A. Sá, C. Rodrigues Neto, C.; R. R. Rosa. Journal of Geophysical Research, 107, D20, 30-1-30-7 (2002).

[8] L. F. Burlaga, A. F.-Viñas. Geophysical Research Letters, 31L16807, doi:10.1029/2004GL020715 (2004).

[9] M. J. A. Bolzan, Y. Sahai, P. R. Fagundes, R. R. Rosa, F. M. Ramos, and J. R. Abalde. Journal of Atmospheric SolarTerrestrial Physics, (2005) in press.

[10] U. Frisch. Turbulence, Cambridge University Press, Cambridge, (1995), $296 \mathrm{pp}$.

[11] J. C. Wyngaard and J. C. Weil. Physics of Fluids A, 3(1), 155 (1991).

[12] N. V. Antonov, J. Honkonen, A. Mazzino, and P. MuratoreGinanneschi, Phys. Rev. E, 62, 5 R5891-R5894, (2000).

[13] L. F. Burlaga, A. Forman, Miriam, Journal of Geophysical Research (Space Physics), 107A11, SSH 18-1, (2002).
[14] Y. Meyer, Ondelettes - Ondelettes et opérateurs. I. Hermann, Paris, (1990), $215 \mathrm{p}$.

[15] W. Gao and B. L. Li, Journal of Applied Meteorology, 3211, 1717 (1993).

[16] I. Daubechies. Ten lectures on wavelets, SIAM, (1992), p. 278-285.

[17] H. Y. Weng, K. M. Lau, Journal of the Atmospheric Sciences, 5117, 2523 (1994)

[18] M. Farge, N. Kevlahan, V. Perrier, and E. Goirand. Wavelets and turbulence. Proceedings of the IEEE, 844, 639 (1996).

[19] MTOF/PM Data by Carrington Rotation. http://umtof.umd.edu/pm/crn/, (2004).

[20] A. N. Kolmogorov. C. R. Acad. Sci., USSR 30, 301 (1941).

[21] F. M. Ramos, R. R. Rosa, C. Rodrigues Neto, M. J. A. Bolzan, and L. D. A. Sá . Nonlinear Analysis, 47, 3521 (2001).

[22] R. Bruno, V. Carbone, L. Sorriso-Valvo, and B. Bavassano. Radial evolution of solar wind intermittency in the inner heliosphere. astro-ph/0303578, 2003.

[23] F. M. Ramos, M. J. A. Bolzan, R. R. Rosa, and L. D. A. Sa. Physica D - Nonlinear Phenomena, 193, 278 (2004).

[24] D. Biskamp, E. Schwarz. Physics of Plasmas, 8 7, 3282 (2001).

[25] G.-M. Le, J.-L. Wang. Chinese Journal of Astronomical and Astrophysical, 3, 391 (2003).

[26] J. K. Hargreaves. The Upper Atmosphere and Solar-Terrestrial Relations, (1979).

[27] G. G. Katul, M. B. Parlange, C. R. Chu, Physics of Fluids, 67, 2480 (1994). 